Recepción: 15 / 04 / 2017

Ciencias Administrativas

Aceptación: 01 / 05 / 2017

Articulo Científico

Publicación: 15 / 05 / 2017

\title{
Evaluación del sistema de control interno aplicado en los procesos de una
} cooperativa de ahorro y crédito

Evaluation of the internal control system applied in the processes of a credit union

Avaliação do sistema de controlo interno aplicados nos processos de uma cooperativa de crédito

Washington A. Camacho-Villota

wcamacho@utb.edu.ec

Alex V. Toapanta-Suntaxi ${ }^{\text {" }}$ atoapanta@utb.edu.ec

Darwin J. Gil-Espinoza "' dgil@utb.edu.ec

Correspondencia: wcamacho@utb.edu.ec

Magister en Contabilidad y Auditoría; Contador Público Auditor; Universidad Técnica de Babahoyo, Ecuador.

Magister en Administración de Empresas; Diploma Superior en Docencia Universitaria; Licenciado en Ciencias de la Educación Especialización Informática Educativa; Universidad Técnica de Babahoyo, Ecuador.

III. Magister en Contabilidad y Auditoría; Contador Público Auditor; Universidad Técnica de Babahoyo, Ecuador. 


\section{Resumen}

El Control Interno dentro de una institución comprende la organización sistemática del trabajo administrativo y de los procedimientos de rutina, con el objeto de prevenir el fraude y errores. Las finalidades del control interno son principalmente disuasivas, preventivas y, en última instancia, correctoras. Entonces, el Control Interno es una herramienta surgida para disminuir significativamente los riesgos en las cuales se haya afectada la institución.

El tema de la tesis es la evaluación del sistema de control interno aplicado en los procesos de la cooperativa de ahorro y crédito Vinces Ltda.", mediante el método de cuestionarios aplicados a las diferentes áreas de la organización, se quiere establecer el nivel de confianza y consecuentemente el riesgo institucional.

El área de operaciones de una Institución de créditos es la encargada de la ejecución de la metodología y parte de las estrategias de crecimiento. Desde el año 1963, la institución ha experimentado un fuerte crecimiento en sus operaciones, la estructura del área se ha visto delimitada ya que su cumplimiento no se ha llevado cabalmente, debido a esta falta de control.

La evaluación del sistema de control interno tiene como propósito disminuir el nivel de fraudes y desfalcos de fondos en la institución por falta de control en sus actividades de operaciones, lo cual puede ser superado mediante la aplicación de una serie de recomendaciones que contribuyan a mejorar y perfeccionar sus actividades operacionales.

Palabras clave: Sistema de control; cooperativa; fraude. 


\begin{abstract}
Internal Control within an institution involves the systematic organization of administrative work and routine procedures, in order to prevent fraud and errors. The purposes of internal control are primarily dissuasive, preventive and, ultimately, corrective. Internal Control is then a tool that has emerged to significantly reduce the risks in which the institution has been affected.
\end{abstract}

The subject of the thesis is the evaluation of the internal control system applied in the processes of the credit union Vinces Ltda. ", Through the method of questionnaires applied to the different areas of the organization, we want to establish the level of trust And consequently institutional risk.

The area of operations of a credit institution is in charge of the execution of the methodology and part of the growth strategies. Since 1963, the institution has experienced strong growth in its operations, the structure of the area has been delimited since its compliance has not been fully implemented due to this lack of control.

The evaluation of the internal control system aims to reduce the level of fraud and embezzlement of funds in the institution due to lack of control in its operations activities, which can be overcome by applying a series of recommendations that contribute to improve and Improve its operational activities.

Key words: Control system; cooperative; fraud. 


\section{Resumo}

O Controle Interno dentro de uma instituição envolve a organização sistemática de trabalhos administrativos e procedimentos de rotina, a fim de evitar fraudes e erros. Os objetivos do controle interno são principalmente dissuasivos, preventivos e, em última instância, corretivos. Controle interno é então uma ferramenta que emergiu para reduzir significativamente os riscos em que a instituição foi afetada.

O tema da tese é a avaliação do sistema de controle interno aplicado nos processos da cooperativa Vinces Ltda. ", Através do método de questionários aplicados nas diferentes áreas da organização, queremos estabelecer o nível de confiança e, conseqüentemente, o risco institucional.

A área de atuação de uma instituição de crédito é responsável pela execução da metodologia e parte das estratégias de crescimento. Desde 1963, a instituição experimentou um forte crescimento em suas operações, a estrutura da área foi delimitada, uma vez que sua conformidade não foi totalmente implementada devido a essa falta de controle.

A avaliação do sistema de controle interno visa reduzir o nível de fraude e desfalque de fundos na instituição por falta de controle nas atividades de operações, o que pode ser superado pela aplicação de uma série de recomendações que contribuem para melhorar e melhorar suas atividades operacionais.

Palavras chave: Sistema de controlo; cooperativa; fraude. 


\section{Introducción.}

El Control Interno comprende la organización sistemática del trabajo administrativo y de los procedimientos de rutina, con el objeto de prevenir el fraude y errores. Las finalidades del control interno son principalmente disuasivas, preventivas y, en última instancia, correctoras.

Lo importante es determinar y seleccionar los ingresos de información que se registran al sistema a fin de que mediante dicho proceso, podamos obtener una información que verdaderamente sirva como producto final a los intereses de una organización. Por ello, al evaluar el sistema de Control Interno implantado por la Cooperativa de Ahorro y Crédito Vinces Ltda., para el desarrollo de sus operaciones, beneficiará directamente a los asociados, directivos, empleados y clientes de la institución.

Los resultados de la evaluación condujeron a una mejor organización, se establecieron líneas claras de responsabilidad; permitieron satisfacer las necesidades y expectativas de los clientes brindándoles un servicio eficiente, efectivo, confiable, seguro y flexible, dirigido a conseguir los más altos niveles de calidad y excelencia y orientando las actividades hacia la mejora continua.

Existen una serie de herramientas que permiten mejorar una organización, he optado por una de ellas, la evaluación del Sistema de Control Interno, que incremente el desarrollo estructurado de la cooperativa, y esto nos permitirá contar con nuestras propias experiencias sobre la organización, la temática tratada y los aspectos legales que encierra este enriquecedor tema.

Con esta investigación entregamos a la institución una serie de recomendaciones que permitieron organizar y dirigir las actividades administrativas y financieras de la cooperativa, maximizar los recursos que posee, asegurando además en todo momento la operatividad y 
confiabilidad de sus actividades, con ello se incrementó el capital social de sus socios, se brindó un mejor servicios y se aseguró el funcionamiento.

La presente investigación tiene como objetivo evaluar el sistema de control interno y su influencia en los procesos de la Cooperativa de Ahorro y Crédito Vinces Ltda.

\section{Materiales y métodos.}

En esta investigación se va a utilizar los siguientes métodos:

\section{Empíricos:}

Método de Observación: Este método nos permitirá observar el desarrollo de los procedimientos, actividades y tareas que realizan el personal de la institución.

Encuestas: En esta investigación se utilizará una Guía para la Evaluación del Sistema de Control Interno. La misma que nos servirá para determinar a cabalidad si se está llevando un control adecuado en las funciones que se realizan en concordancia con los manuales de la institución.

Análisis Documental: Se realizará una revisión de los documentos que se obtendrán como medio de verificación a las respuestas dadas en la encuesta realizada mediante la aplicación de la Guía de Control Interno.

\section{Métodos Teóricos}

En este método se realizará el análisis y síntesis de los instrumentos aplicados para determinar las principales irregularidades que se presentan en el sistema de control interno del área de operaciones. 


\section{Inductivo y Deductivo}

Este método nos ayudará para determinar los principales elementos que se van a incorporar en la investigación.

\section{Productos obtenidos}

Evaluación del Sistema de Control Interno y propuesta de mejoras para el perfeccionamiento de la estructura organizacional y funciones de las diferentes dependencias, en especial del área de operaciones de la Cooperativa.

Novedad y aportes teóricos prácticos de la investigación

En el siguiente trabajo de investigación presento una propuesta de mejoras para el perfeccionamiento de la estructura organizacional y funciones de las diferentes dependencias, en especial del área de operaciones de la Cooperativa, el mismo que se obtendrá aplicando las herramientas de control interno basado en el Sistema COSO.

\section{Resultados.}

\section{Encuestas}

En esta investigación se va a aplicar la técnica de encuestas, utilizando una guía de evaluación del sistema de control interno, el mismo que se basará en la modalidad del Sistema Coso.

La estructura del control interno, propuesta por el modelo COSO, identifica cinco componentes interrelacionados y son los siguientes: 
Ambiente de Control

Evaluación de Riesgos

Actividades de Control

Supervisión o Monitoreo

Con la aplicación del cuestionario de control interno determinamos el nivel de confianza y de riesgos que existe en los procesos que tiene la entidad para cumplir los objetivos institucionales.

El cuestionario de control interno será aplicado al Gerente General, Presidente y al Jefe de Operaciones de la Cooperativa, ya que ellos son los encargados de supervisar y verificar que se cumplan con los procesos establecidos en el manual de Funciones de la institución.

En la guía para evaluar el Sistema de Control Interno se utilizaron preguntas cerradas, de respuestas SI y NO, a las cuales se les dieron los siguientes valores:

\begin{tabular}{|c|c|}
\hline RESPUESTA & CALIFICACIÓN \\
\hline SI & 1 \\
\hline NO & 0 \\
\hline
\end{tabular}

\section{Encuesta}

\section{Guía para la evaluación del sistema de control interno}

ENTIDAD:

Área o rubro evaluado:

Período:

Norma Técnica aplicable:

Objetivo:
Cooperativa de Ahorro y Crédito Vinces Ltda.

Departamento de Operaciones

Del 1 al 30 de enero de 2013

200 - AMBIENTE DE CONTROL

Verificar que la máxima autoridad haya establecido en forma clara y por escrito líneas de conducta y medidas de control para alcanzar los objetivos de la institución. 


\section{NCI 200-01 - INTEGRIDAD Y VALORES ÉTICOS}

\section{RESULTADO DE LA APLICACIÓN DE LA NORMA TÉCNICA:}

Paran obtener los resultados de esta norma de control, hemos utilizado una matriz de calificación del nivel de confianza y riesgo global.

Las preguntas fueron contestadas por el Gerente General, en la cual obtuvimos una calificación de 1, porque las respuestas que nos dio el funcionario de la institución fueron positivas.

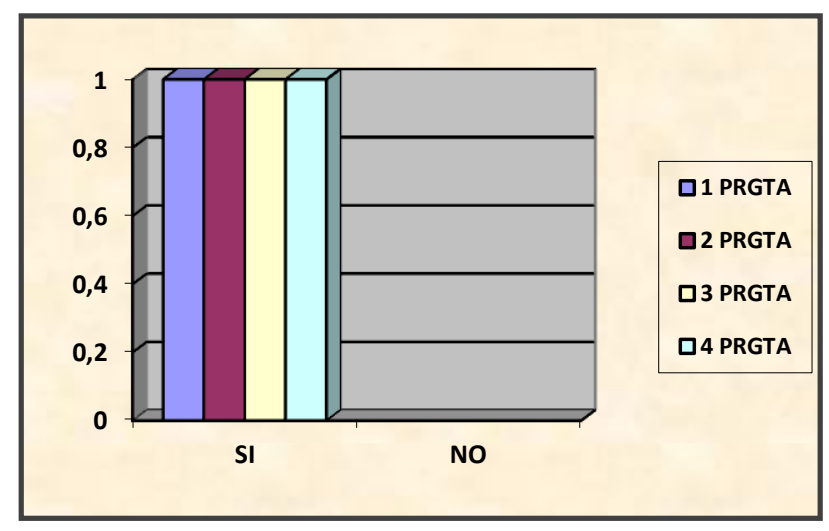

Observamos que en la Norma Integridad y Valores éticos, la institución si cuenta con un código de ética debidamente aprobado por la máxima autoridad.

\section{NCI 200-02 - ADMINISTRACIÓN ESTRATÉGICA}

\section{RESULTADO DE LA APLICACIÓN DE LA NORMA TÉCNICA:}

Paran obtener los resultados de la NCI 200-02, hemos utilizado una matriz de calificación del nivel de confianza y riesgo global. 
Las preguntas fueron contestadas por el Oficial de Monitoreo, en la cual obtuvimos una calificación de 1, aunque las respuestas fueron diversas que nos dio el funcionario de la institución la mayoría fueron positivas.

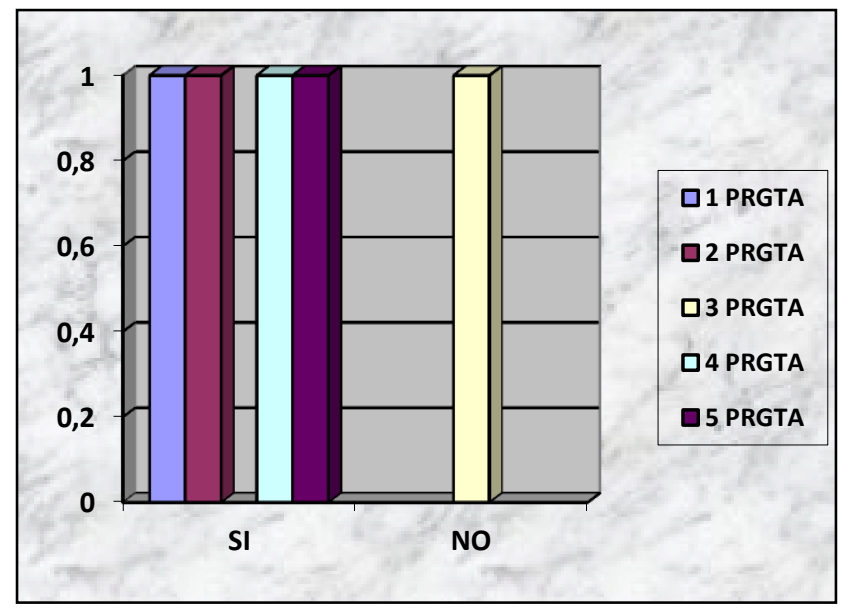

Observamos que en la Norma Administración Estratégica, se cumple con lo establecido en el sistema de planificación anual que realiza la institución.

\section{CONCLUSIÓN: NORMA TECNICA APLICABLE 200 - AMBIENTE DE CONTROL}

Se utilizó una Matriz de Calificación del Nivel de Confianza y riesgo Global, en donde encontramos la siguiente calificación, para los niveles de confianza y de riesgo:

\begin{tabular}{|c|c|c|}
\hline \multicolumn{3}{|c|}{ NIVEL DE CONFIANZA } \\
\hline BAJO & MODERADO & ALTO \\
\hline $15 \%-50 \%$ & $51 \%-75 \%$ & $76 \%-95 \%$ \\
\hline $85 \%-50 \%$ & $49 \%-25 \%$ & $24 \%-5 \%$ \\
\hline ALTO & MODERADO & BAJO \\
\hline NIVEL DE RIESGO (100 - NC)
\end{tabular}


Donde obtuvimos los siguientes resultados de las NCI 200 - 01 Y NCI 200-02

\begin{tabular}{|c|c|c|c|}
\hline CT & $\mathbf{8}$ & RIESGO & ENFOQUE \\
\hline PT & 9 & & \\
\hline NC & $89 \%$ & ALTO & \\
RI & $11 \%$ & BAJO & CUMPLIMIENTO \\
\hline
\end{tabular}

\section{Dónde:}

CT: Es el número de preguntas que obtuvieron calificación 1

PT: Número de preguntas utilizadas en la NCI

NC: $89 \%$ (Se obtiene de la división de CT y PT)

RI: $11 \%$ (Es la resta del $100 \%$ y el NC)

Revisando la tabla de calificación de Nivel de Confianza, observamos que el Nivel de Confianza en esta NCI es Moderado (89\%) y su Nivel Riesgo es Moderado (11\%), dándole un enfoque de. Cumplimiento.

\section{GUÍA PARA LA EVALUACIÓN DEL SISTEMA DE CONTROL INTERNO}

ENTIDAD:

Área o rubro evaluado:

Período:

Norma Técnica aplicable:

Objetivo:
Cooperativa de Ahorro y Crédito Vinces Ltda.

Departamento de Operaciones

Del 1 al 30 de enero de 2013

\section{0 - EVALUACIÓN DEL RIESGO}

Verificar si la institución realiza la identificación y valoración del riesgo para obtener la suficiente información acerca de situaciones con probabilidad de ocurrencia, que afecten el logro de sus objetivos institucionales. 


\section{NCI 300-01 - IDENTIFICACIÓN DE RIESGOS}

\section{RESULTADO DE LA APLICACIÓN DE LA NORMA TÉCNICA:}

Paran obtener los resultados de la NCI 300-01, hemos utilizado una matriz de calificación del nivel de confianza y riesgo global.

Las preguntas fueron contestadas por el Oficial de Monitoreo, en la cual obtuvimos diversas calificaciones, porque hubieron respuestas positivas y negativas.

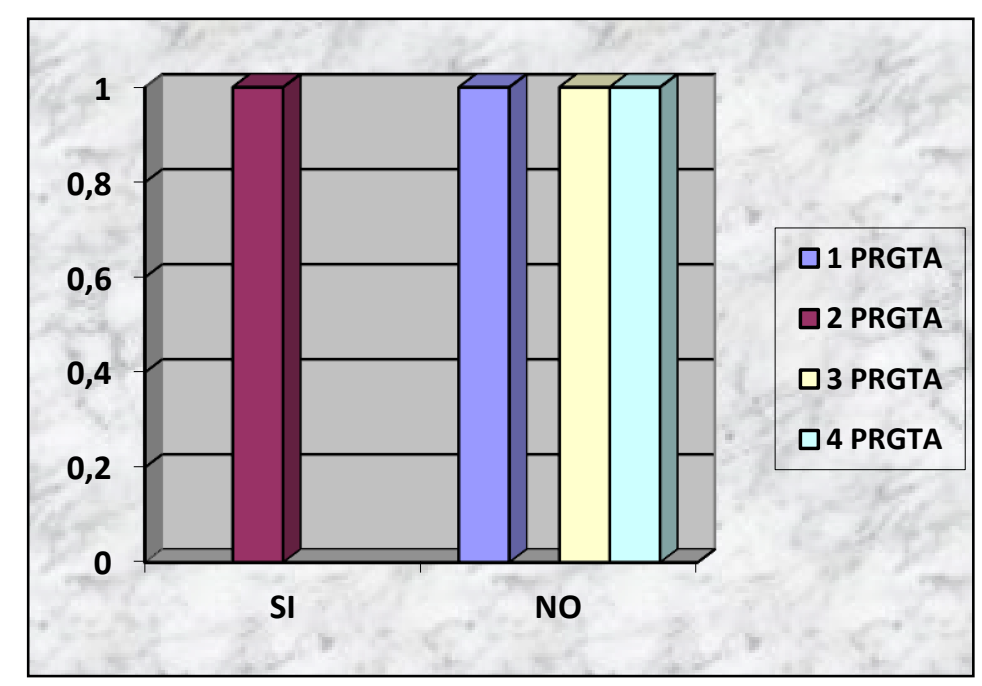

Observamos que la institución no cuenta con un mapa de riesgos institucional, pero al mismo tiempo los directivos de la misma identifican los riesgos que pueden afectar el logro de los objetivos institucionales.

\section{NCI 300-03 - VALORACIÓN DE LOS RIESGOS}

\section{RESULTADO DE LA APLICACIÓN DE LA NORMA TÉCNICA:}

Paran obtener los resultados de la NCI 300-03, hemos utilizado una matriz de calificación del nivel de confianza y riesgo global. 
Las preguntas fueron contestadas por el Gerente General, en la cual obtuvimos diversas calificaciones, porque hubo respuestas positivas y negativas.

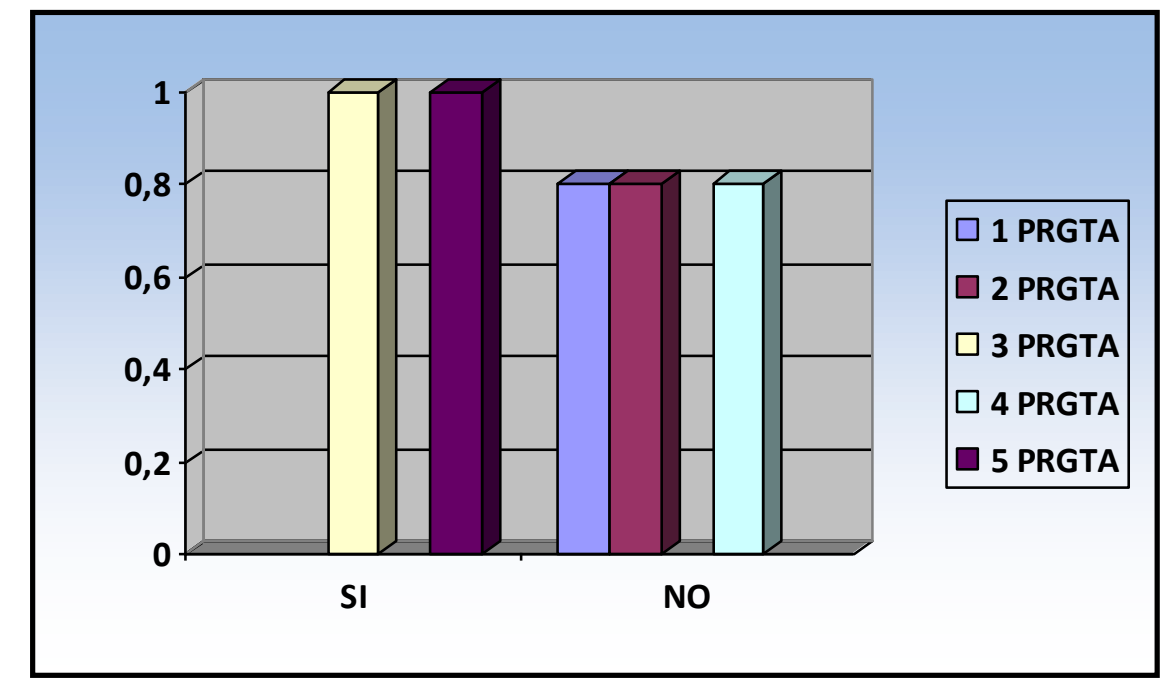

Observamos que la institución no cuenta con una valoración de riesgos, pero al mismo tiempo en la institución identifican riesgos potenciales que puedan interferir en la consecución de los objetivos.

\section{CONCLUSIÓN: NORMA TECNICA APLICABLE 300 - EVALUACIÓN DE RIESGOS}

Se utilizó una Matriz de Calificación del Nivel de Confianza y riesgo Global, en donde encontramos la siguiente calificación, para los niveles de confianza y de riesgo:

\begin{tabular}{|l|l|l|}
\hline \multicolumn{3}{|c|}{ NIVEL DE CONFIANZA } \\
\hline BAJO & MODERADO & ALTO \\
\hline $15 \%-50 \%$ & $51 \%-75 \%$ & $76 \%-95 \%$ \\
\hline $85 \%-50 \%$ & $49 \%-25 \%$ & $24 \%-5 \%$ \\
\hline ALTO & MODERADO & BAJO \\
\hline NIVEL DE RIESGO (100 - NC) \\
\hline
\end{tabular}


Donde obtuvimos los siguientes resultados de las NCI 300 - 01, NCI 300 - 03

\begin{tabular}{|l|l|l|l|}
\hline CT & 3 & RIESGO & ENFOQUE \\
\hline PT & 9 & & \\
\hline NC & $33 \%$ & BAJO & \\
\hline RI & $67 \%$ & ALTO & SUSTANTIVO \\
\hline
\end{tabular}

\section{Donde:}

CT: Es el número de preguntas que obtuvieron calificación 1

PT: Número de preguntas utilizadas en la NCI

NC: 33\% (Se obtiene de la división de CT y PT)

RI: $67 \%$ (Es la resta del 100\% y el NC)

Revisando la tabla de calificación de Nivel de Confianza, observamos que el Nivel de Confianza en esta NCI es Bajo (33\%) y su Nivel Riesgo es Alto (67\%), dándole un enfoque Sustantivo.

\section{GUÍA PARA LA EVALUACIÓN DEL SISTEMA DE CONTROL INTERNO}

ENTIDAD:

Área o rubro evaluado:

Período:

Norma Técnica aplicable:

Objetivo:
Cooperativa de Ahorro y Crédito Vinces Ltda.

Departamento de Operaciones

Del 1 al 30 de enero de 2013

400 - ACTIVIDADES DE CONTROL

Verificar si la institución realiza actividades de control en todos los niveles y funciones, que estén relacionadas directamente con los objetivos de la entidad. 


\section{NCI 401-02 - AUTORIZACIÓN Y APROBACIÓN DE TRANSACCIONES Y}

\section{OPERACIONES}

\section{RESULTADO DE LA APLICACIÓN DE LA NORMA TÉCNICA:}

Paran obtener los resultados de la NCI 401-02, hemos utilizado una matriz de calificación del nivel de confianza y riesgo global.

Las preguntas fueron contestadas por el Presidente de la Institución, en la cual obtuvimos calificación de 1, porque las respuestas que nos dio el funcionario de la institución fueron positivas.

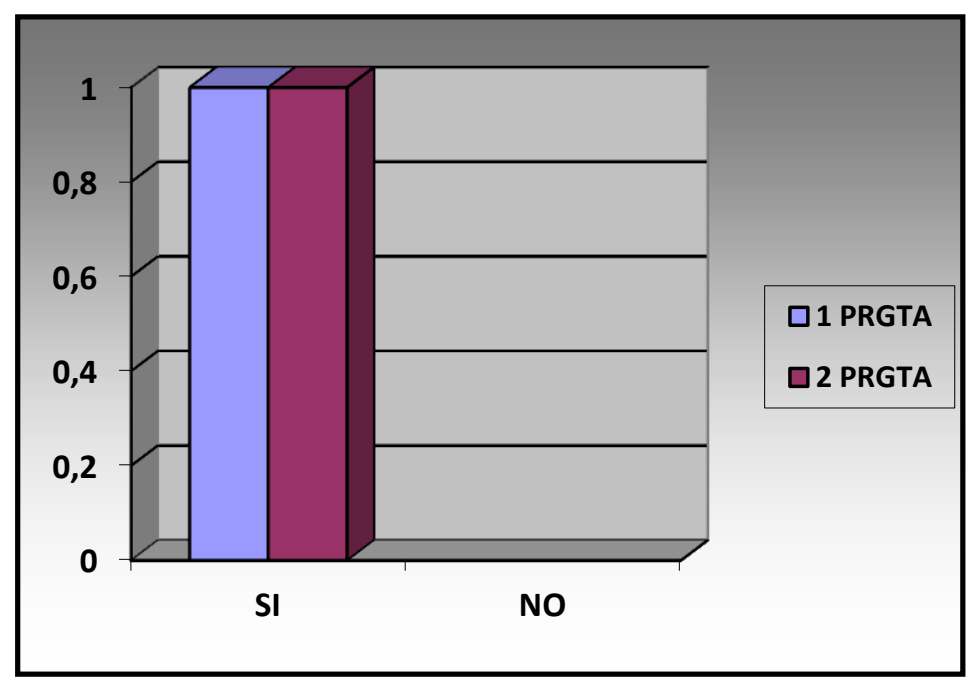

Observamos que la Máxima autoridad ha establecido procedimientos de autorización de ejecución de procesos de control, los mismos que son difundidos a sus servidores. 


\section{NCI 401-03 - SUPERVISIÓN}

\section{RESULTADO DE LA APLICACIÓN DE LA NORMA TÉCNICA:}

Paran obtener los resultados de la NCI 401-03, hemos utilizado una matriz de calificación del nivel de confianza y riesgo global.

Las preguntas fueron contestadas por el Presidente de la Institución, en la cual obtuvimos calificación de 1, porque las respuestas que nos dio el funcionario de la institución fueron positivas.

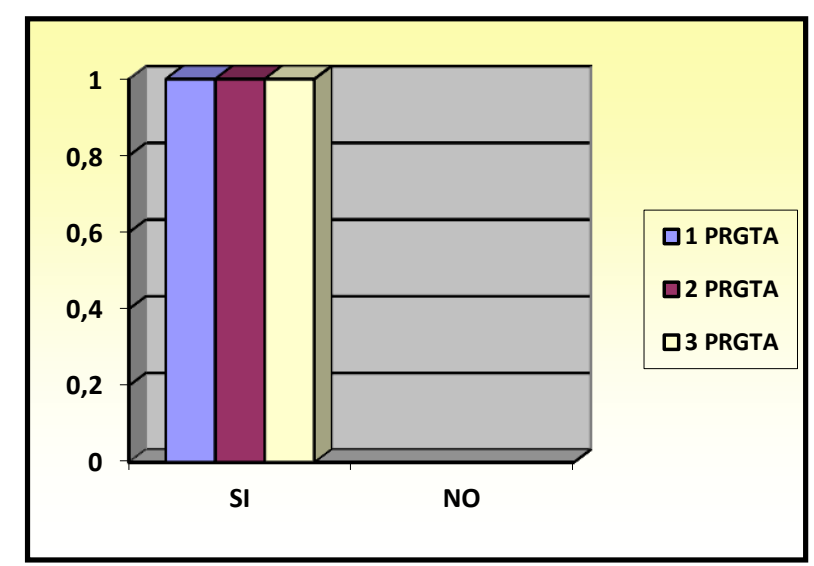

Observamos que los directivos de la entidad, han establecido procedimientos de supervisión de los procesos y operaciones, los procesos que se los realiza constantemente para asegurar que las operaciones se desarrollen de acuerdo a lo establecido en las políticas de la institución.

\section{NCI 401-03 - 01 SUPERVISIÓN DEL ÁREA DE OPERACIONES}

\section{RESULTADO DE LA APLICACIÓN DE LA NORMA TÉCNICA:}

Paran obtener los resultados de la NCI 401-03- 01, hemos utilizado una matriz de calificación del nivel de confianza y riesgo global. 
Las preguntas fueron contestadas por el Gerente General, en la cual obtuvimos calificación de 1, aunque las respuestas que nos dio el funcionario de la institución fueron diversas la mayoría fueron positivas.

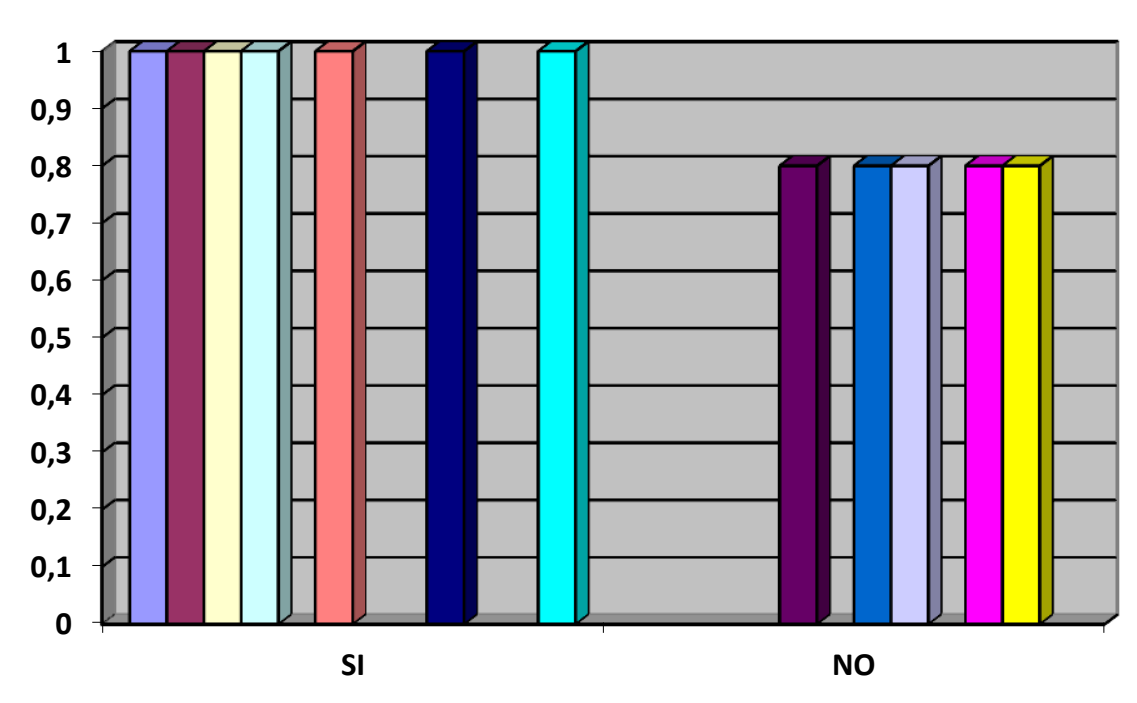

\begin{tabular}{|l|}
\hline$\square 1$ PRGTA \\
$\square 2$ PRGTA \\
$\square 3$ PRGTA \\
$\square 4$ PRGTA \\
$\square 5$ PRGTA \\
$\square 6$ PRGTA \\
$\square 7$ PRGTA \\
$\square 8$ PRGTA \\
$\square 9$ PRGTA \\
$\square 10$ PRGTA \\
$\square 11$ PRGTA \\
$\square 12$ PRGTA \\
\hline
\end{tabular}

Podemos observar que los procesos que tienen el área de operaciones no son muy adecuados para llevar un buen control de las actividades que realizan en las oficinas.

\section{NCI 402 ADMINISTRACIÓN FINANCIERA}

\section{2-02 -CONTROL PREVIO AL COMPROMISO}

\section{RESULTADO DE LA APLICACIÓN DE LA NORMA TÉCNICA:}

Paran obtener los resultados de la NCI 402-02, hemos utilizado una matriz de calificación del nivel de confianza y riesgo global.

Las preguntas fueron contestadas por el Gerente General, en la cual obtuvimos calificación de 1, porque las respuestas que nos dio el funcionario de la institución fueron positivas. 


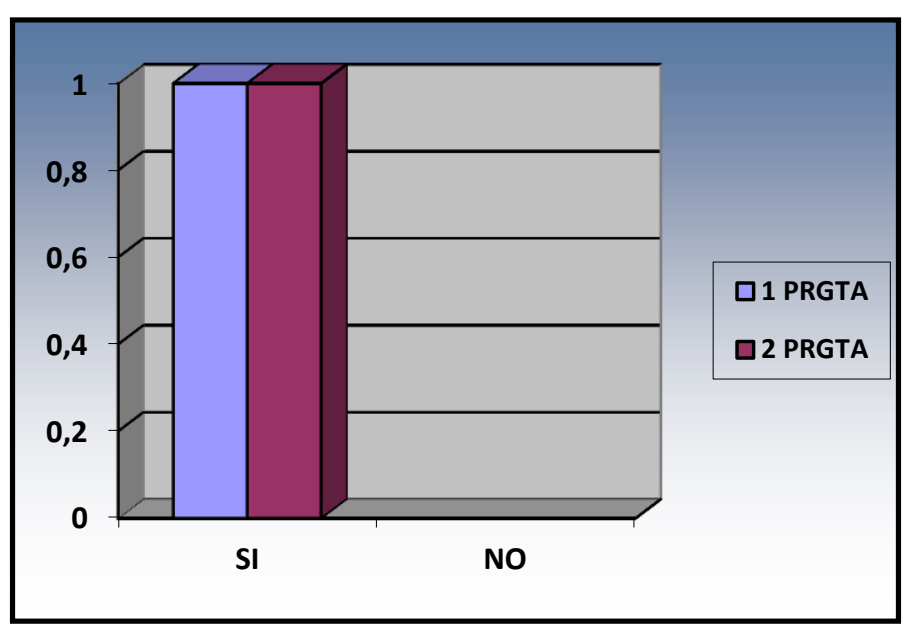

Observamos que la operación financiera de la institución está directamente relacionada con la misión de la entidad, y a su vez reúne los requisitos legales pertinentes para que pueda llevarlas a cabo.

\section{NCI 402-04 - CONTROL DE LA EVALUACIÓN EN LA EJECUCIÓN DEL PRESUPUESTO POR RESULTADOS}

\section{RESULTADO DE LA APLICACIÓN DE LA NORMA TÉCNICA:}

Paran obtener los resultados de la NCI 402-04, hemos utilizado una matriz de calificación del nivel de confianza y riesgo global.

Las preguntas fueron contestadas por el Gerente General, en la cual obtuvimos calificación de 1, porque las respuestas que nos dio el funcionario de la institución fueron positivas. 


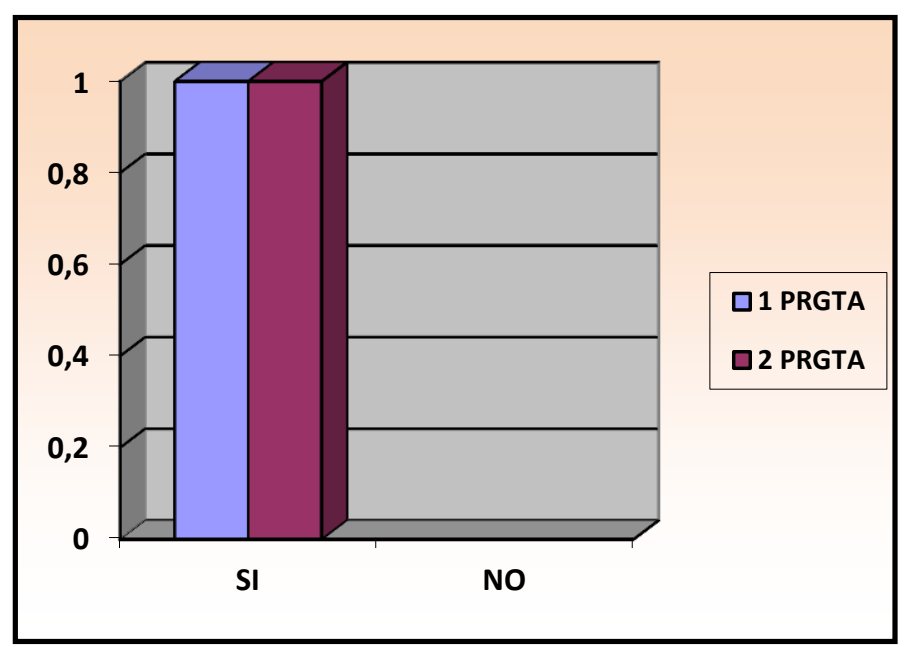

Observamos que la Máxima autoridad dispone que los responsables de la ejecución presupuestaria efectúen el seguimiento y evaluación del cumplimiento de los objetivos.

\section{NCI 403 - ADMINISTRACION FINANCIERA}

\section{3 - 01 DETERMINACIÓN Y RECAUDACIÓN DE LOS INGRESOS}

\section{RESULTADO DE LA APLICACIÓN DE LA NORMA TÉCNICA:}

Paran obtener los resultados de esta norma hemos utilizado una matriz de calificación del nivel de confianza y riesgo global.

Las preguntas fueron contestadas por el Oficial de Monitoreo, en la cual obtuvimos calificación de 1, porque las respuestas que nos dio el funcionario de la institución fueron positivas.

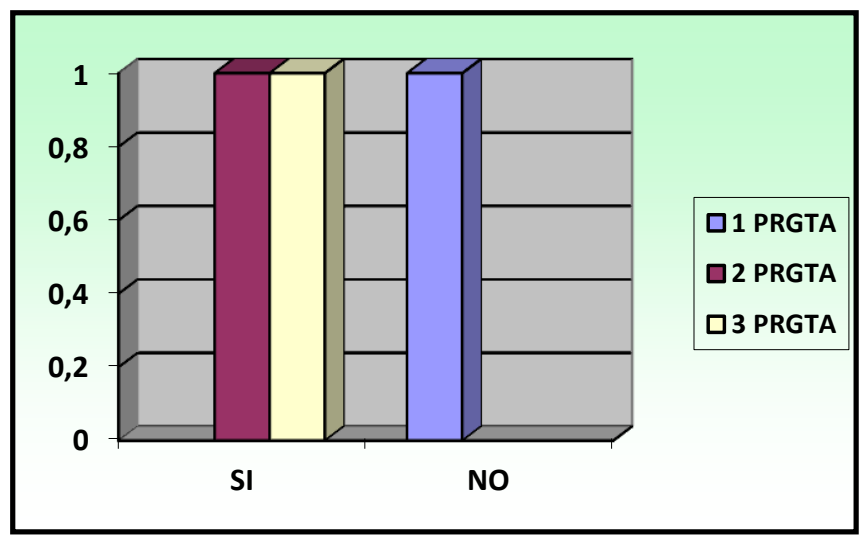


Observamos que los ingresos que recauda la institución por recuperación de los créditos otorgados y depósitos en cuentas se realizan solo en la ventanilla de la Institución y no se realizan mediante depósitos directos por los socios en cuentas corrientes de la institución, y se realiza el respectivo depósito al día siguiente para disminuir el riesgo de tener dinero en efectivo.

\section{NCI 403 - 02 CONSTANCIA DOCUMENTAL DE LA RECAUDACIÓN}

\section{RESULTADO DE LA APLICACIÓN DE LA NORMA TÉCNICA:}

Paran obtener los resultados de la NCI 403-02, hemos utilizado una matriz de calificación del nivel de confianza y riesgo global.

Las preguntas fueron contestadas por el Gerente General, en la cual obtuvimos una calificación de 1 , porque hubo respuestas positivas.

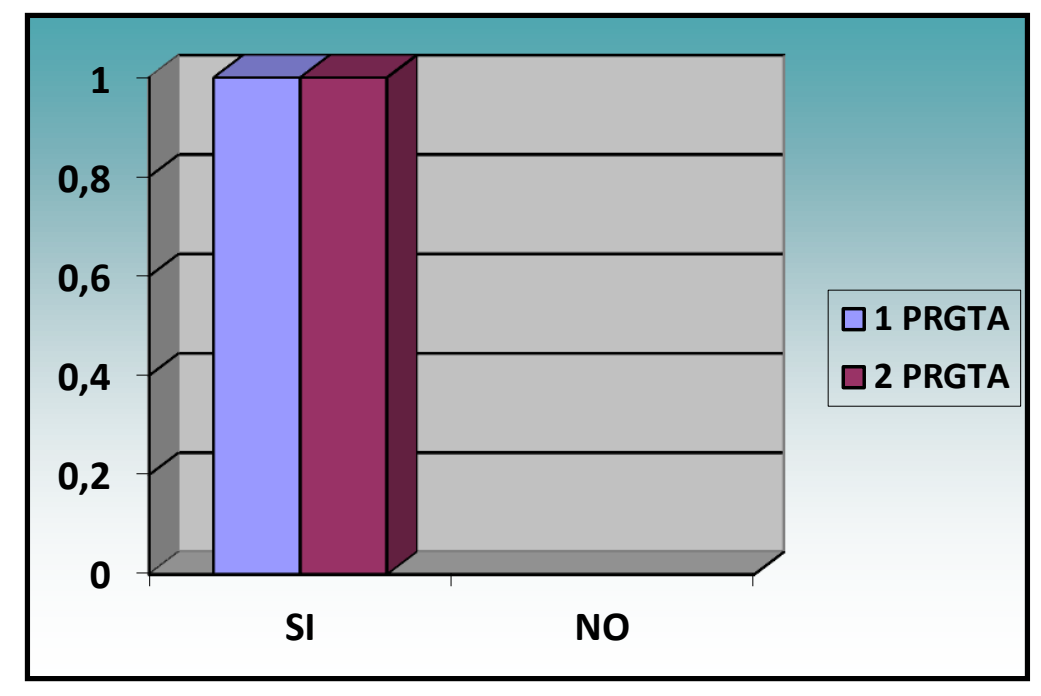

Observamos que en la Institución se han establecido normas acerca de la recolección de dinero en efectivo, donde se debe entregar al cliente un comprobante de ingreso que certifica que el cliente ha entregado el dinero en efectivo, el cual se está cumpliendo. 


\section{NCI 403 - 04 VERIFICACION DE LOS INGRESOS}

\section{RESULTADO DE LA APLICACIÓN DE LA NORMA TÉCNICA:}

Paran obtener los resultados de la NCI 403-04, hemos utilizado una matriz de calificación del nivel de confianza y riesgo global.

Las preguntas fueron contestadas por el Gerente General, en la cual obtuvimos una calificación de 1, porque hubo respuestas positivas.

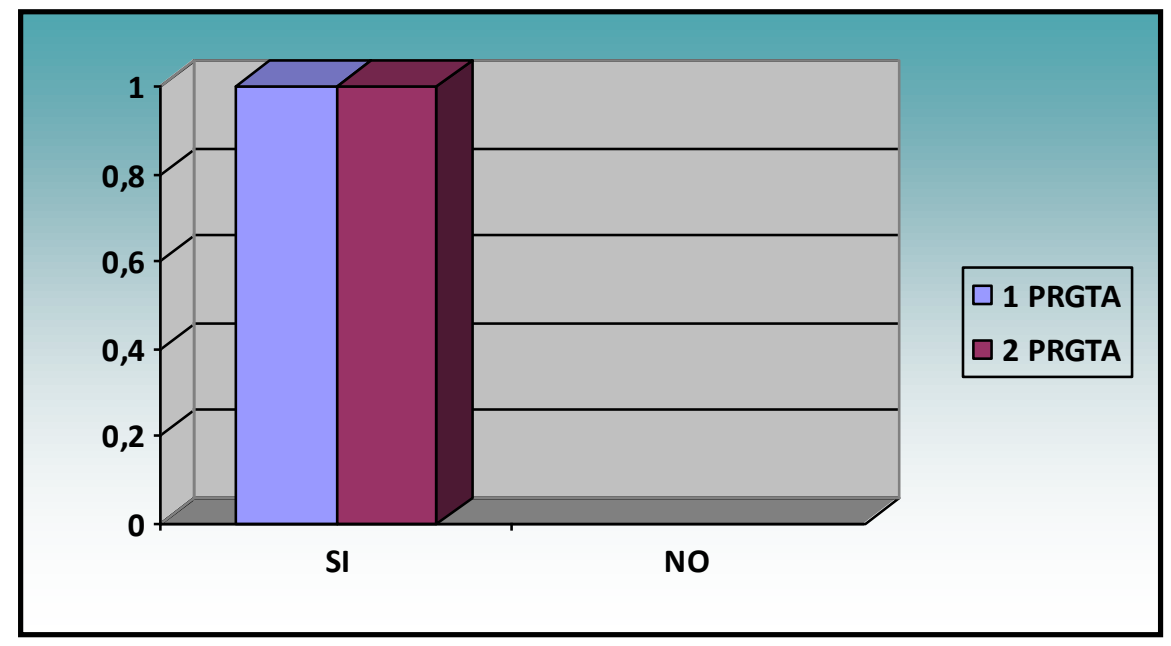

Observamos que en la Institución se han establecido normas acerca de la recolección de dinero en efectivo y el depósito en las cuentas de la Institución, donde el depósito se debe de realizar hasta antes de las 11 h00 del día siguiente y de esta forma dar cumplimiento a lo establecidos en la norma. 


\section{CONCLUSIÓN: NORMA TECNICA APLICABLE 400 - ACTIVIDADES DE CONTROL}

Se utilizó una Matriz de Calificación del Nivel de Confianza y riesgo Global, en donde encontramos la siguiente calificación, para los niveles de confianza y de riesgo:

\begin{tabular}{|l|l|l|}
\hline \multicolumn{3}{|c|}{ NIVEL DE CONFIANZA } \\
\hline BAJO & MODERADO & ALTO \\
\hline $15 \%-50 \%$ & $51 \%-75 \%$ & $76 \%-95 \%$ \\
\hline $85 \%-50 \%$ & $49 \%-25 \%$ & $24 \%-5 \%$ \\
\hline ALTO & MODERADO & BAJO \\
\hline NIVEL DE RIESGO (100 - NC) \\
\hline
\end{tabular}

Donde obtuvimos los siguientes resultados de las NCI 400 - 01, NCI 401 - 02, NCI 401 03, NCI $401-03$ - 01, NCI 402 - 02, NCI 402 - 04, NCI 403 - 01, NCI 403 - 02, NCI 403 - 04

\begin{tabular}{|l|l|l|l|}
\hline CT & 21 & RIESGO & ENFOQUE \\
\hline PT & 28 & & \\
\hline NC & $75 \%$ & MODERADO & \\
\hline RI & $25 \%$ & MODERADO & $\begin{array}{l}\text { MIXTO-DOBLE } \\
\text { PROPÓSITO }\end{array}$ \\
\hline
\end{tabular}

\section{Dónde:}

CT: Es el número de preguntas que obtuvieron calificación 1

PT: Número de preguntas utilizadas en la NCI

NC: 75\% (Se obtiene de la división de CT y PT)

RI: $25 \%$ (Es la resta del $100 \%$ y el NC)

Revisando la tabla de calificación de Nivel de Confianza, observamos que el Nivel de Confianza en esta NCI es Moderado (75\%) y su Nivel Riesgo es Moderado (25\%), dándole un enfoque de Mixto - Doble propósito, no obstante habiendo obtenido respuestas positivas que no están generando un buen control en las actividades. 


\section{GUÍA PARA LA EVALUACIÓN DEL SISTEMA DE CONTROL INTERNO PARA LAS}

\section{UNIDADES DE OPERACIONES DE UNA COOPERATIVA DE AHORRO Y CREDITO}

ENTIDAD:

Área o rubro evaluado:

Período:

Norma Técnica aplicable:

Objetivo:

\section{COOPERATIVA DE AHORRO Y CREDITO} VINCES LTDA.

Departamento de Operaciones

Del 1 al 30 de ENERO de 2013

600 - SEGUIMIENTO

Verificar si la máxima autoriza y los directivos de la entidad establecen procedimientos de seguimiento continuo, evaluaciones periódicas o una combinación de ambas para asegurar la eficacia del sistema de control interno.

\section{NCI 600 - SEGUIMIENTO}

\section{0-01 - SEGUIMIENTO CONTINUO O EN OPERACIÓN}

\section{RESUMEN DE NORMA TÉCNICA APLICABLE:}

Paran obtener los resultados de la NCI 600, hemos utilizado una matriz de calificación del nivel de confianza y riesgo global.

Las preguntas fueron contestadas por el Gerente General, en la cual obtuvimos diversas calificaciones, porque hubo respuestas positivas y negativas.

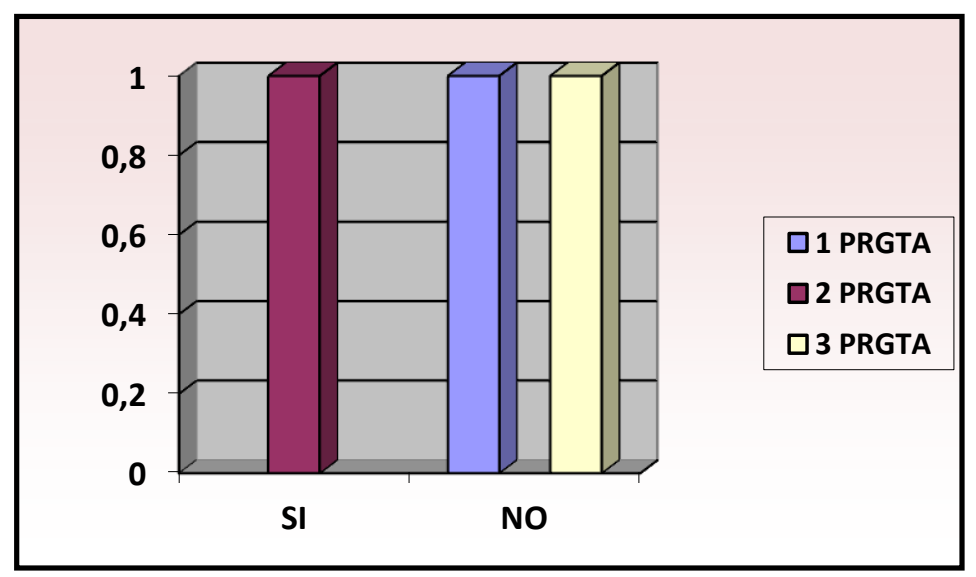


Observamos que los directivos de la entidad realizan seguimientos continuos de las actividades y gestiones que realiza el personal en sus tareas, pero al mismo tiempo la máxima autoridad no realiza seguimientos del ambiente externo.

\section{NCI 600-02 - EVALUACIONES PERIÓDICAS}

\section{RESULTADO DE LA APLICACIÓN DE LA NORMA TÉCNICA:}

Paran obtener los resultados de la NCI 600-02, hemos utilizado una matriz de calificación del nivel de confianza y riesgo global.

Las preguntas fueron contestadas por el Gerente General, en la cual obtuvimos diversas calificaciones, porque hubo respuestas positivas y negativas.

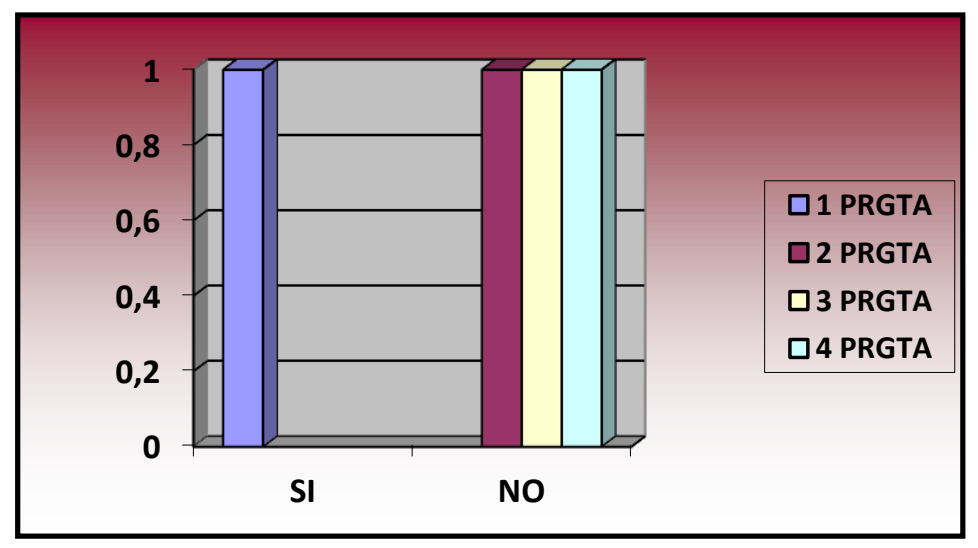

\section{CONCLUSIÓN: NORMA TECNICA APLICABLE 600 - SEGUIMIENTO}

Se utilizó una Matriz de Calificación del Nivel de Confianza y riesgo Global, en donde encontramos la siguiente calificación, para los niveles de confianza y de riesgo: 


\begin{tabular}{|l|l|l|}
\hline \multicolumn{3}{|c|}{ NIVEL DE CONFIANZA } \\
\hline BAJO & MODERADO & ALTO \\
\hline $15 \%-50 \%$ & $51 \%-75 \%$ & $76 \%-95 \%$ \\
\hline $85 \%-50 \%$ & $49 \%-25 \%$ & $24 \%-5 \%$ \\
\hline ALTO & MODERADO & BAJO \\
\hline NIVEL DE RIESGO (100 - NC) \\
\hline
\end{tabular}

Donde obtuvimos los siguientes resultados de las NCI 600, NCI $600-02$.

\begin{tabular}{|l|l|l|l|}
\hline CT & $\mathbf{2}$ & RIESGO & ENFOQUE \\
\hline PT & 7 & & \\
\hline NC & $29 \%$ & BAJO & \\
\hline RI & $71 \%$ & ALTO & SUSTANTIVO \\
\hline
\end{tabular}

\section{Dónde:}

CT: Es el número de preguntas que obtuvieron calificación 1

PT: Número de preguntas utilizadas en la NCI

NC: 29\% (Se obtiene de la división de CT y PT)

RI: $71 \%$ (Es la resta del 100\% y el NC)

Revisando la tabla de calificación de Nivel de Confianza, observamos que el Nivel de Confianza en esta NCI es Bajo (29\%) y su Nivel Riesgo es Alto (71\%), dándole un enfoque Sustantivo.

\section{ANÁLISIS DE DOCUMENTACION DE SOPORTE A FIN DE VERIFICAR RESULTADOS DE ENTREVISTA DE EVALUACION DEL CONTROL INTERNO:}

Una vez aplicada la guía de evaluación del sistema de control interno a los funcionarios del área de operaciones de la institución antes mencionada, procedemos a verificar las repuestas obtenidas. 
Para tal efecto se otorga una calificación a cada una de las preguntas elaboradas en la guía de evaluación según la norma técnica de auditoría aplicada, dicha calificación será asignada luego de realizar el análisis documental y constatar la veracidad de la respuesta otorgada a la pregunta, teniendo como respaldo la información documental obtenida en el proceso de investigación.

A continuación se muestra un ejemplo de cómo se obtienen los valores de las calificaciones que fueron aplicadas en las normas técnicas de control interno

NCI. 200 Ambiente de Control

\begin{tabular}{|c|l|l|}
\hline $\mathbf{N}^{\mathbf{0}}$ Nivel & Niveles & Calificación \\
\hline 1. & Incipiente & 2.2 \\
\hline 2. & Básico & 4.4 \\
\hline 3. & Confiable & 6.7 \\
\hline 4. & Muy Confiable & 8.9 \\
\hline 5. & Óptimo & 11.1 \\
\hline
\end{tabular}

Donde los valores de la calificación los obtuvimos aplicando la siguiente fórmula:

\section{Nivel Incipiente}

- Valor óptimo $=100 \%$

- Número de preguntas de norma técnica aplicada $=9$ preguntas

- $\quad$ Total de Niveles $=5$

- $\quad$ Número de Nivel $=1$ 
Valor óptimo $=$ Resultado

\# preg

$\frac{100}{9}=11,111$

$\underline{\text { Resultado }}=$ Resultado

\# Niveles

$\underline{11,111}=2,22$

5

Resultado (número de nivel)

2,22 (1) = 2,2 Valor Nivel Incipiente

\section{Nivel Básico}

Valor óptimo $=100 \%$

Número de preguntas de norma técnica aplicada $=9$ preguntas

Total de Niveles $=5$

Numero de nivel $=2$

$\underline{\text { Valor óptimo }}=$ Resultado \# preg

$\frac{100}{9}=11,111$

$\underline{\text { Resultado }}=$ Resultado

\# Niveles

$\frac{11,111}{5}=2,22$

Resultado (número de nivel)

2,22 (2) = 4,4 Nivel Básico

\section{Nivel Confiable}

Valor óptimo $=100 \%$

Número de preguntas de norma técnica aplicada $=9$ preguntas

Total de Niveles $=5$

Número de nivel $=3$ 
Valor óptimo $=$ Resultado \# preg

$$
\frac{100}{9}=11,111
$$

$\underline{\text { Resultado }}=$ Resultado

\# Niveles

$$
\frac{11,111}{5}=2,22
$$

Resultado (número de nivel)

$$
\text { 2,22 (3) = 6,7 Nivel Confiable }
$$

\section{Nivel Muy Confiable}

Valor óptimo $=100 \%$

Número de preguntas de norma técnica aplicada $=9$ preguntas

Total de Niveles $=5$

Número de Nivel $=4$

Valor óptimo $=$ Resultado \# preg

$\frac{100}{9}=11,111$

$\underline{\text { Resultado }}=$ Resultado

\# Niveles

$$
\frac{11,111}{5}=2,22
$$

Resultado (Número de Nivel)

\section{2,22 (4) = 8,09 Nivel Muy Confiable}

\section{Nivel Óptimo}

Valor óptimo $=100 \%$

Número de preguntas de norma técnica aplicada $=9$ preguntas

Total de Niveles $=5$

Número de nivel $=5$ 
$\underline{\text { Valor óptimo }}=$ Resultado

\# preg

$\frac{100}{9}=11,111$

$\underline{\text { Resultado }}=$ Resultado

\# Niveles

$\frac{11,111}{5}=2,2$

Resultado (número de nivel)

$2,22(5)=\mathbf{1 1 , 1}$ Nivel Óptimo

Una vez que hemos obtenido los valores de las calificaciones procedemos a calificar las preguntas que fueron realizadas en la encuesta.

\section{Conclusiones.}

El control interno constituye una parte fundamental en toda organización ya que de este depende que todas las operaciones se realicen adecuadamente y que los riesgos existentes puedan ser manejados y disminuidos.

La evaluación efectuada al sistema de Control Interno de la Cooperativa de Ahorro y Crédito, determina que esta presenta algunas deficiencias e incumplimientos de documentación necesaria para desarrollar adecuadas actividades de control tales como: falta de cumplimiento en las políticas establecidas por la institución, falta de seguimiento en las actividades de control; falta de documentación de soportes de egreso, entre otras.

La carencia de un adecuado control interno ha generado que se presente irregularidades, así como no permitir que la organización crezca organizadamente y logre productos de calidad.

Determinadas las falencias por falta de control interno se pudo recomendar el plan de mejoras a las políticas y procedimientos, el mismo que será implementado con el fin de mejorar las actividades de desarrollo de la Cooperativa; ya que se alcanzaría orientación en las políticas de créditos, en los procedimientos de diferentes actividades salvaguardando los recursos de la 
institución. Además que permitirá poner en práctica el control interno, el mismo que será muy beneficioso porque otorgará seguridad razonable en el cumplimiento de los objetivos cuyos riesgos de cometer errores serán minimizados, prevenidos, corregidos, detectados a tiempo, disminuyendo su impacto y probabilidad de ocurrencia.

\section{Recomendaciones.}

Considerando que en todas las organizaciones es una necesidad fundamental contar con Manuales de Políticas y Procedimientos, los que permitan controlar el cumplimiento de actividades, reducir errores; es por estas necesidades que se recomienda:

Se implemente el presente Plan de Mejoras a las políticas y procedimientos, a fin de que se convierta en una guía útil para poder realizar actividades adecuadamente observando los procedimientos a seguir, llevando controles efectivos.

Se recomienda que el presente Plan de Mejoras sea monitoreado y evaluado periódicamente por la dirección administrativa a fin de verificar el cumplimiento de los controles internos y vigilar el desempeño de sus Colaboradores.

Debe aplicarse exámenes especiales en las preguntas efectuadas en la encuesta de Guía de Evaluación de Control Interno y obtuvimos como resultado actividades de nivel incipiente y básico.

La Cooperativa debe aplicar la evaluación de control existente, no solo por parte de auditoría interna y externa, sino como un proceso integral en las operaciones, sobre todo cuando se implementan, para que desde el principio se efectúen de forma correcta con el adecuado manejo de los riesgos existentes. 
La Cooperativa debe contar con personal capacitado para que efectúe las evaluaciones de los controles existentes así como la identificación de los riesgos a los que enfrenta la misma, para que estos riesgos sean disminuidos.

\section{Bibliografía.}

Ambrosio, Juárez, V. (2008). Auditoría Práctica de Estados Financieros. Guatemala: Editorial Lessa.

Cano, D. (2011). Contra el Fraude. Buenos Aires: Ediciones Granica C.A.

Coopers \& Lybrand. (1997). Los Nuevos Conceptos del Control Interno - Informe COSO. Madrid: Ediciones Díaz de Santos S.A.

Dorta, Velázquez, J. (2005). La Evaluación de Riesgos como Componente básico del Sistema de Control Interno. México: Editorial Thomson.

Estupiñan, Gaitán, R. (2009). Análisis de Informe Coso I y II. Colombia: Ecoe Ediciones.

Fernández, Fernández, M. (2003). El control, Fundamento de la Gestión por procesos y la calidad total. (2a Edición). Madrid: Editorial Esic.

Fonseca, Luna, O. (2011). Sistema de Control Interno para organizaciones, (1era. Ed). Lima.

Instituto de Auditores. (2010). Guía para la Supervisión de Sistemas de Control Interno. Ecuador: Autor.

Mantilla, Samuel. \& Cante, Sandra. (2005). Auditoria de Control Interno. Bogotá: Coe Ediciones.

Mejía, García, B. (2006). Gerencia de Procesos para la Organización y el Control Interno. Bogotá. Ecoe Ediciones.

Moreno, Olvera, L. (2004). Auditoría 1. Fundamentos de Auditoría. México: Editorial Thomson.

Paniagua, Bravo, V. \& Paniagua, Pinto, M. (2004). El Contralor. (3era. Edición). México: Editorial Thomson.

Peña, Bermúdez, J. (2007). Control, Auditoría y Revisión Fiscal Bogotá: Ecoe Ediciones Ltda.

Perdomo, Moreno, A. (2004). Fundamentos de Control Interno. México: Thomson Editorial.

Santillana, González, J. (2003). Establecimientos de Sistemas de Control Interno. México. Editorial Thomson.

Spencer, Pickett, K. (2007). Manual Básico de Auditoría Interna. Barcelona: Ediciones Gestión 2000.

Warren, Carl. Y Reeve, James. (2005). Contabilidad Financiera. (9na. Edición). México: Editorial Thomson. 